\title{
MÉTODO ANALÍTICO PARA DETERMINAÇÃO DOS CORANTES AURAMINA EM TESTES DE ECOTOXICIDADE E EM ÁGUAS SUPERFICIAIS
}

\author{
Carina C. J. Azevedo ${ }^{a}$, Marcia K. Kasahara ${ }^{\mathrm{b}}$ e Cassiana C. Montagnera,b,*,(])
}

${ }^{a}$ Faculdade de Tecnologia, Universidade Estadual de Campinas, 13484-332 Limeira - SP, Brasil

bDepartamento de Química Analítica, Instituto de Química, Universidade Estadual de Campinas, 13083-970 Campinas - SP, Brasil

Recebido em 21/12/2020; aceito em 02/03/2021; publicado na web em 24/03/2021

\begin{abstract}
ANALYTICAL METHOD FOR DETERMINATION OF AURAMINE DYES IN ECOTOXICITY TESTS AND IN SURFACE WATERS. Dyes can be considered important environmental contaminants. Auramine dyes, known commercially as Solvent Yellow 34 and Basic Yellow 2, are used in several industrial products, including textile and paper. In this study, an analytical method was developed and validated using Liquid-Liquid Extraction and High-Performance Liquid Chromatography coupled to Diode Array Detector (LLE and HPLC-DAD) to determine the exposure concentrations in the ecotoxicological tests with auramine dyes, increasing the results reliability about the hazard of these compounds. The method was also used to monitor auramine dyes in surface water samples, in a region considered one of the most critical regions for dyes contamination in Brazil. The results showed no risk for the aquatic organisms considering the analyzed samples. The method using simple instrumentation showed the results of selectivity, linearity ( $\left.\mathrm{R}^{2}>0.99\right)$, precision ( $\mathrm{CV}$ : 5.6-29\%) and accuracy (recovery: 82-90\%) considered satisfactory for the two purposes with Instrumental LOQ of the $12 \mu \mathrm{g} \mathrm{L} \mathrm{L}^{-1}$ and the Method LOQ of the $12 \mathrm{ng} \mathrm{L}^{-1}$. The results indicated that the proposed method was effective and practical for the auramine determination in environmental levels and will be an important tool to verify the potential risk of auramine dyes in future studies.
\end{abstract}

Keywords: HPLC-DAD; surface water; environmental monitoring; ecotoxicity test; risk assessment.

\section{INTRODUÇÃO}

Corantes são substâncias utilizadas em diversos segmentos industriais, tais como, têxteis, farmacêuticos, de cosméticos, alimentícias, de papel, entre outros. Existem mais de 100.000 corantes disponíveis comercialmente e cerca de 1 milhão de toneladas de corantes são produzidos todos os anos. ${ }^{1,2}$ Com ampla produção e utilização industrial, as perdas também são relevantes, cerca de $1 \%$ a $2 \%$ desses corantes são perdidos durante o processo de produção, que podem envolver até 500 etapas de sínteses complexas. ${ }^{2}$ Além disso, em indústrias têxteis, que são os maiores consumidores de corantes, responsáveis por dois terços da produção total, estima-se que aproximadamente $10 \%$ dos corantes são perdidos na etapa de tintura. ${ }^{2,3}$ Corantes já foram detectados em águas superficiais, localizadas próximas a descargas de efluentes de indústrias têxteis em diferentes lugares do mundo, tais como Brasil, ${ }^{4-8}$ Canadá ${ }^{\text {e Índia. }}{ }^{10}$

Classificar um corante pela sua estrutura química permite identificá-los rapidamente por estarem agrupados em categorias que possuem características físico-químicas semelhantes. Diferentes classes podem ser encontradas, tais como azo, antraquinona, indigoide, ftalocianinas, metina e polimetinas, etc. ${ }^{1,2}$ Dentre as diversas classes, há os corantes difenilmetanos, que são geralmente agrupados aos triarilmetanos. Esses corantes alcançaram considerável importância na economia e são amplamente utilizados devido à sua versatilidade. ${ }^{11}$ Corantes triarilmetanos, como Malachite Grenn e Crystal Violet já foram detectados em efluentes de indústrias têxteis e de papel, bem como em águas superficiais. ${ }^{12}$ Em relação aos corantes difenilmetanos, os representantes mais importantes são o Michler's hydrol e a auramina. ${ }^{11}$ Entretanto, não há trabalhos na literatura que investiguem a ocorrência destes corantes em compartimentos ambientais.

*e-mail: ccmonta@unicamp.br
Os corantes auramina (Solvent Yellow 34 e Basic Yellow 2, também conhecidos como Auramina e Auramina O) (Figura 1), são utilizados no tingimento de couro, juta (fibra têxtil vegetal), algodão e papel..$^{13,14}$ Estes corantes são altamente tóxicos para os organismos aquáticos e o Predicted Non-Effect Concentrations (PNEC) para ambas formas químicas possuem valores considerados ambientalmente relevantes, de $0,92 \mu \mathrm{g} \mathrm{L}^{-1}$ (Solvent Yellow 34) e 4,6 $\mathrm{gg} \mathrm{L}^{-1}$ (Basic Yellow 2). ${ }^{15}$ Embora seja diferente a ecotoxicidade para cada corante, trata-se de um ácido fraco $(\mathrm{pKa}=10,12)$ e as concentrações das espécies individuais são dependentes do $\mathrm{pH}$ do meio. Desta forma, espera-se que sua forma iônica seja predominante em águas superficiais.

Quantificar a ocorrência destes compostos em águas é se suma importância para verificar se há um potencial risco para os organismos aquáticos e, dessa forma, investir em tecnologias de tratamento.

Uma abordagem para avaliar o risco ecotoxicológico dos contaminantes ambientais, incluindo os corantes, relaciona o perigo e a exposição, comparando a concentração medida em um determinado compartimento, conhecida internacionalmente como MEC (Measured Environmental Concentration), com um critério para proteção da vida aquática, conhecido internacionalmente como PNEC. Esses critérios são concentrações consideradas seguras nas quais não se espera efeitos adversos para a biota aquática se expostos durante toda a sua vida. Nesta abordagem um coeficiente de risco conhecido internacionalmente como RQ (Risk Quotient) é obtido dividindo MEC por PNEC, onde valores de RQ menores que 1 indicam que o composto não impõe risco ambiental. ${ }^{16,17}$

Esse tipo de avaliação só é possível quando se tem métodos analíticos com precisão e detectabilidade adequadas, ou seja, menores que os PNEC adotados, para realizar o monitoramento dos contaminantes nos compartimentos ambientais, de tal forma que os dados possam subsidiar as avaliações de risco de forma mais conclusiva. ${ }^{16,17}$

Nesse contexto, o desenvolvimento de métodos analíticos para quantificação dos contaminantes de interesse ambiental é 
a)

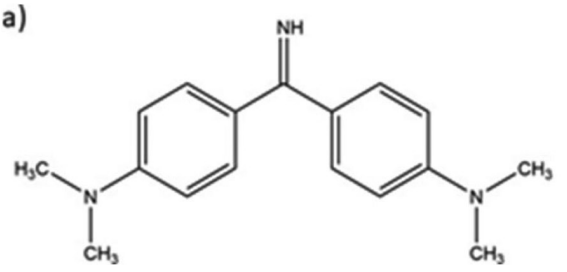

b)<smiles>CN(C)c1ccc(C(=N)c2ccc(N(C)C)cc2)cc1</smiles>

Figura 1. Estruturas químicas dos corantes (a) Solvent Yellow 34 ou Auramina $\left(\mathrm{C}_{17} \mathrm{H}_{21} \mathrm{~N}_{3}\right)$ e (b) Basic Yellow 2 ou Auramina O ( $\mathrm{C}_{17} \mathrm{H}_{21} \mathrm{~N}_{3} \mathrm{HCl}$ )

importante também para os ecotoxicologistas. Uma vez que para aumentar a confiabilidade do ensaio, é necessário quantificar o(s) contaminante(s) nas soluções utilizadas nos testes de ecotoxicidade, no qual, as concentrações nominais só poderão ser utilizadas para expressar os resultados dos testes quando as concentrações de exposição (quantificadas através dos métodos analíticos) estiverem dentro do intervalo de $80-120 \%$ das nominais. ${ }^{18,19}$ Entretanto, são escassos os trabalhos na literatura que reportam as quantificações das concentrações reais dos corantes em testes de ecotoxicidade, muito devido às dificuldades em se obter métodos analíticos validados que possam ser empregados para esse fim.

Um exemplo pode ser relatado com o trabalho de Parrott e Bartlett (2016) que empregaram SPE-HPLC/DAD (Solid Phase Extraction e High Performance Liquid Cromatography coupled to Diode Array Detector) para quantificar quatro corantes, Disperse Yellow 7, Sudan Red G, Acid Blue 80 e Acid Blue 129 em amostras de testes toxicológicos com o peixe da espécie Pimephales promelas em concentrações entre 10 e $10.000 \mu \mathrm{g} \mathrm{L}^{-1}$. Os autores relataram que algumas concentrações de exposição não estavam dentro do intervalo aceitável entre 80 a $120 \%$ das concentrações nominais. ${ }^{20}$ No entanto, parâmetros de validação importantes como a exatidão do método, medida pelo ensaio de recuperação dos analitos, por exemplo, não foram reportados no artigo, não sendo, portanto, possível concluir se a dispersão dos resultados obtidos na quantificação dos corantes nas amostras foi devido à baixa \% de recuperação do método analítico empregado ou ainda devido à degradação dos corantes durante os testes ecotoxicológicos. ${ }^{20}$

Diante desse cenário, desenvolver um método analítico com sensibilidade adequada para medir as concentrações de exposição sem a necessidade de um preparo de amostra seria essencial e, assim, evitaria as possíveis perdas ocasionadas na etapa de extração dos analitos de interesse. Entretanto, dependendo das concentrações testadas, a etapa de concentração dos analitos é imprescindível para obter um método com detectabilidade adequada para a análise. Nesse caso, o analista deve garantir que a metodologia utilizada apresente exatidão e precisão adequadas de forma a não interferir no resultado final da análise química.

Nesse contexto, o objetivo deste trabalho foi desenvolver e validar um método analítico utilizando HPLC/DAD para a quantificação dos corantes auramina com sensibilidade adequada para a aplicação tanto na aferição de concentrações nominais dos testes ecotoxicológicos, quanto na determinação dos corantes em amostras de águas superficiais. Considerando que os corantes auramina são utilizados em diversas atividades industriais e, possivelmente, podem atingir os diferentes compartimentos ambientais, dentre eles, os corpos d'água superficiais, os resultados obtidos nesse trabalho serão ferramentas essenciais para subsidiar futuras avaliações de risco destinadas à proteção da vida aquática.

\section{PARTE EXPERIMENTAL}

\section{Reagentes}

Padrões analíticos dos corantes auramina, Solvent Yellow 34
(CAS number 492-80-8, pureza de 97\%) e Basic Yellow 2 (CAS number 2465-27-2, pureza de 87\%), foram adquiridos da Sigma Aldrich $^{\circledR}$. Os solventes, metanol (Tedia ${ }^{\circledR}$ ), ácido fórmico (Fluka Analytical $^{\circledR}$ ), diclorometano $\left(\right.$ Tedia $\left.^{\circledR}\right)$, acetonitrila (Sigma-Aldrich ${ }^{\circledR}$ ), hexano (95\% Tedia ${ }^{\circledR}$ ) possuíam grau HPLC para análise de resíduos. Os sais utilizados também foram de alta pureza: cloreto de sódio (99\% Synth ${ }^{\circledR}$ ), sulfato de magnésio (98-102\% Dinâmica ${ }^{\circledR}$ ) e acetato de amônio (97\% Synth $\left.^{\circledR}\right)$. A água foi ultrapurificada utilizando um deionizador modelo Synergy Water Purification System, da Synergy ${ }^{\circledR}$ com resistividade de $18 \mathrm{M} \Omega \cdot \mathrm{cm}$ a $25{ }^{\circ} \mathrm{C}$. As soluções estoque (300 $\mathrm{mg} \mathrm{L}^{-1}$ ) foram preparadas dissolvendo a massa quantitativamente pesada dos padrões sólidos em $10 \mathrm{~mL}$ de metanol. As soluções de trabalho em diferentes concentrações foram preparadas a partir de diluições sucessivas da solução estoque em uma mistura de água e solvente orgânico de composição semelhante à fase móvel empregada na separação cromatográfica.

\section{Análise por HPLC/DAD}

A análise cromatográfica foi realizada em um equipamento Shimadzu, modelo SCL10AVP equipado com uma coluna de guarda Shim-pack G-ODS(4) octadecil de 4 mm de diâmetro interno, marca Shimadzu e uma coluna de separação de fase reversa Shiseido Co. modelo CAPCLLE PAK C18 AG120 S-5 $\mu \mathrm{m}$, de $250 \mathrm{~mm}$ de comprimento e 4,6 $\mathrm{mm}$ de diâmetro interno, acoplado com detector de ultravioleta por arranjo de diodos modelo SPD- M10VP, Shimadzu.

Para o desenvolvimento do método analítico alguns parâmetros foram analisados a fim de se obter as melhores condições cromatográficas de análise, tais como: composição da fase móvel (FM), vazão da fase móvel (0,7; 0,8 e 0,9 $\left.\mathrm{mL} \mathrm{min}^{-1}\right)$ e tipo de eluição (gradiente ou isocrático). A escolha dos melhores parâmetros para o método cromatográfico foi realizada com base na comparação dos diferentes cromatogramas obtidos em $437 \mathrm{~nm}$, que corresponde ao comprimento de onda de máxima absorção para os corantes analisados. Para definição de qual solvente utilizar na composição da fase móvel, foram avaliados os cromatogramas obtidos com Metanol/ Água e Acetonitrila/Água. Posteriormente, a água foi acidificada com ácido fórmico $(0,1 \%)$. O tipo de eluição foi determinado observando a forma do pico e o tempo de retenção, optando pelo qual obteve um menor tempo de corrida (o que proporciona maior rapidez para a análise) e, também, aquele que apresentou o pico mais simétrico possível (evitando a presença de caudas). Em todas as medidas o volume de injeção foi de $20 \mu \mathrm{L}$. Os solventes orgânicos e a água utilizados como FM foram filtrados por um sistema de filtração a vácuo utilizando filtros de nylon de $0,22 \mu \mathrm{m}$ (Whatman ${ }^{\circledR}$ ) para os solventes orgânicos e membranas de papel (Sartorius Stedim Boitech®) com 0,22 $\mu \mathrm{m}$ para os solventes aquosos.

\section{Preparo de amostra}

Para o preparo de amostras foram propostos dois métodos de extração: (i) extração em fase sólida (SPE, do inglês Solid Phase Extraction) e (ii) extração líquido-líquido (LLE, do inglês LiquidLiquid Extraction), com o objetivo de avaliar o melhor resultado 
para exatidão do método, a qual foi medida pela porcentagem de recuperação dos analitos estudados. Para tal, replicatas de alíquotas (400 mL) contendo misturas dos padrões dos analitos em três níveis de concentrações $\left(0,5 ; 1,0\right.$ e $\left.5,0 \mu \mathrm{g} \mathrm{L}^{-1}\right)$ foram extraídas em triplicata por ambos os métodos e analisadas por HPLC/DAD.

\section{Extração em fase sólida (SPE)}

Para SPE foi utilizado o cartucho Oasis HLB (Waters) contendo $500 \mathrm{mg}$ de fase sólida e $6 \mathrm{~mL}$ de volume interno. A escolha do sorvente do cartucho foi baseada em Zocolo e colaboradores, ${ }^{6}$ que usaram SPE no preparo de amostras ambientais (águas superficiais e efluentes) para análise de corantes. Além disso, essa é uma fase extratora comumente empregada em análises multiresíduos de contaminantes ambientais devido ao seu amplo espectro de retenção e, portanto, a determinação de auramina poderia ser inserida em métodos já estabelecidos de monitoramento empregando o mesmo preparo de amostras.

Para a extração dos corantes auramina, os cartuchos foram previamente condicionados com $4 \mathrm{~mL}$ de metanol, $4 \mathrm{~mL}$ de água e $4 \mathrm{~mL}$ de acetato de amônio $\left(50 \mathrm{mmol} \mathrm{L}^{-1}\right)$. Um volume de $400 \mathrm{~mL}$ de amostra contendo os corantes em concentrações conhecidas foi extraído a vazão controlada de $7 \mathrm{~mL} \mathrm{~min}^{-1}$, em seguida a remoção dos interferentes foi realizada lavando o cartucho com $4 \mathrm{~mL}$ de solução 1:1 (v/v) acetato de amônio $50 \mathrm{mmol} \mathrm{L}^{-1}$ : metanol. Os analitos de interesse foram eluídos da fase extratora empregando $4 \mathrm{~mL}$ de acetona:metanol $(1: 1 \mathrm{v} / \mathrm{v})$ e $4 \mathrm{~mL}$ de acetona:hexano $(5: 95 \mathrm{v} / \mathrm{v})$. O extrato final foi seco com gás nitrogênio de elevada pureza e os analitos foram ressuspendidos em $1 \mathrm{~mL}$ de solução com composição similar à da fase móvel do método cromatográfico empregado na determinação dos corantes. Finalmente, os extratos foram conservados em freezer a $4{ }^{\circ} \mathrm{C}$ até a análise cromatográfica.

\section{Extração líquido-líquido (LLE)}

A LLE é um método classicamente empregado em análises de corantes. Para a extração dos corantes auramina foi realizado um planejamento experimental para otimizar as condições de análise descritas previamente por Vacchi e colaboradores, ${ }^{7}$ avaliando a proporção de fase extratora, o tempo de agitação, o tempo de extração e a força iônica (necessidade de adicionar cloreto de sódio) (Tabela 1S, Material Suplementar). O método otimizado empregou $400 \mathrm{~mL}$ de amostra e $20 \mathrm{~mL}$ de fase extratora 1:2,5 (v/v) metanol:diclorometano em três alíquotas, as quais foram agitadas por 1,5 min com o tempo de extração estipulado em $5 \mathrm{~min}$, seguido da adição de $\mathrm{MgSO}_{4}$, filtração por gravidade e evaporação da fase extratora, utilizando um evaporador rotativo com temperatura de aproximadamente $40^{\circ} \mathrm{C}$, até restar um volume de $3 \mathrm{~mL}$, sendo então transferido para um tubo de ensaio. Posteriormente, o balão de fundo redondo foi lavado com $3 \mathrm{~mL}$ de fase extratora, e esse volume foi transferido para o mesmo tubo de ensaio contendo a primeira alíquota. Os extratos foram secos com fluxo de nitrogênio de elevada pureza e armazenados sob refrigeração $\left(4^{\circ} \mathrm{C}\right)$ até a análise por HPLC/DAD. Para as análises cromatográficas, os extratos foram ressuspendidos com $1,0 \mathrm{~mL}$ da solução de composição similar a fase móvel empregada na análise instrumental.

\section{Figuras de mérito da validação do método analítico}

O método analítico foi validado de acordo com as recomendações para análise de resíduos, descritas no guia de validação de método disponibilizado pelo Instituto Nacional de Metrologia de $2016^{21}$, seu desempenho foi analisado com base nos resultados obtidos para as seguintes figuras analíticas de mérito: linearidade, faixa de trabalho, limite de detecção instrumental (LDI), limite de quantificação instrumental (LQI), precisão instrumental, seletividade, exatidão, precisão, limites de detecção e quantificação (LD e LQ) do método analítico.

A linearidade foi avaliada por meio de curvas analíticas com padrões contendo diferentes concentrações dos corantes estudados $\left(5,15,25,50,100\right.$ e $\left.200 \mu \mathrm{g} \mathrm{L}^{-1}\right)$, sendo a faixa linear definida como o intervalo entre os limites de quantificação e a maior concentração do padrão analítico empregado a qual se apresentou com um coeficiente de determinação superior a 0,990 .

Os LDI e LQI foram calculados a partir da curva analítica utilizando as equações 1 e 2, respectivamente, conforme recomendações descritas pelo INMETRO e ANVISA..$^{21,22}$

Limite de Detecção $=\frac{3 \times \text { desvio padrão do coeficiente linear da curva }}{\text { coeficiente angular da curva }}$

Limite de Quantificação $=\frac{10 \times \text { desvio padrão do coeficiente linear da curva }}{\text { coeficiente angular da curva }}$

A precisão instrumental foi avaliada em termos de repetibilidade das áreas relativas aos picos cromatográficos obtidos após réplicas de injeção de soluções $(n=6)$ contendo diferentes concentrações dos analitos, dentro da faixa linear de trabalho.

A exatidão e a precisão do método foram avaliadas para os dois preparos de amostras, LLE e SPE, empregando amostras sintéticas preparadas em laboratório, fortificando volumes conhecidos de água ultrapura com 3 diferentes níveis $\left(0,5 ; 1,0\right.$ e $\left.5,0 \mu \mathrm{g} \mathrm{L}^{-1}\right)$ de concentração dos corantes de interesse. A análise foi realizada em triplicata, sendo os resultados expressos em porcentagem de recuperação e coeficiente de variação $(\mathrm{CV})$, respectivamente.

A seletividade do método foi determinada pela comparação dos cromatogramas obtidos após a análise de quatro amostras diferentes: branco (amostra de água ultrapura sem adição dos corantes que passou por todo o processo de extração), amostra sintética (água ultrapura fortificada com os corantes de interesse em concentração de $10 \mu \mathrm{g} \mathrm{L}^{-1}$ a qual passou por todo o processo de preparo de amostras), amostra de água superficial (amostra coletada do Ribeirão das Pedras, Campinas, SP, Brasil) e uma solução padrão (preparada em solução de mesma composição da fase móvel usada na análise cromatográfica contendo os corantes em concentração de $\left.10 \mu \mathrm{g} \mathrm{L}^{-1}\right)$.

Os LD e LQ do método foram calculados conforme o fator de concentração obtido na etapa de preparo de amostras, que pode ser de até 1000 vezes a depender do tipo de amostra analisada.

\section{Aplicação do método}

\section{Testes de ecotoxicidade}

Para validar a utilização do método analítico desenvolvido na quantificação dos corantes auramina em testes de ecotoxicidade, foram analisadas amostras de um teste crônico com o microcrustáceo Daphnia similis. O corante utilizado no ensaio foi o Solvent Yellow 34. O teste foi baseado na OECD n 211 de $2012,{ }^{23}$ no qual foi avaliado a inibição da reprodução a diferentes concentrações do corante durante 14 dias. As concentrações testadas foram 250, 500, 750, 1000, 1500 e $2000 \mu \mathrm{g} \mathrm{L}^{-1}$. O ensaio foi realizado em ambiente com temperatura controlada $\left(21 \pm 1^{\circ} \mathrm{C}\right)$, fotoperíodo de 16 horas de luz e 8 de escuro, com recipientes cobertos, realizando a alimentação a cada 24 horas e a troca total da solução-teste, bem como a contagem do número de neonatas, a cada 48 horas. Ao término do ensaio, somou-se o número de organismos jovens produzidos por réplica. A inibição na reprodução foi o indicativo de toxicidade crônica na amostra. Para a validação do teste, os seguintes parâmetros foram observados nos organismos adultos do controle negativo: a mortalidade não poderia exceder $20 \%$, 
pelo menos $60 \%$ dos organismos apresentarem 3 posturas de filhotes, e produção média de $\geq 23$ neonatas por organismo. As concentrações de exposição $\left(250,500,1000\right.$ e $\left.1500 \mu \mathrm{g} \mathrm{L}^{-1}\right)$ foram avaliadas durante a renovação da solução uma vez por semana, em que uma alíquota foi analisada no dia que as concentrações foram preparadas e outra na troca da solução, após uma exposição de 48 horas.

\section{Amostras ambientais}

O método analítico desenvolvido foi utilizado para avaliar a ocorrência dos corantes auramina em dois rios (Rio Piracicaba e Ribeirão Quilombo) localizados na cidade de Americana, São Paulo, local que possui o maior polo industrial têxtil do Brasil.

No Rio Piracicaba, foram analisadas amostras de dois pontos diferentes. O primeiro foi junto à captação de água da cidade de Americana, à montante do lançamento da Estação de Tratamento de Esgoto (ETE) Carioba e o outro foi a aproximadamente $2 \mathrm{~km}$ a jusante do lançamento da ETE. A ETE Carioba, além de atender as empresas têxteis, também é responsável por $85 \%$ dos esgotos sanitários da cidade de Americana-SP. O sistema de tratamento de efluentes da estação, baseia-se em filtros biológicos aeróbios percoladores e embora os corantes trifenilmetanos possam ser descoloridos ou degradados por meios biológicos, ${ }^{24}$ atualmente a ETE Carioba trabalha com apenas $40 \%$ de eficiência de tratamento em relação a carga orgânica, aumentando as possibilidades de detectar corantes nas águas superficiais. O terceiro ponto de coleta foi no Ribeirão Quilombo, esse corpo hídrico recebe efluentes diretamente de indústrias têxteis da região. Informações referente a localização e as coordenadas geográficas dos pontos amostrais são descritas no Material Suplementar (Figuras 1S e 2S, Tabela 2S). No total, três campanhas foram realizadas e 9 amostras analisadas.

Os três pontos amostrais citados acima estão localizados em uma região com predomínio de indústrias têxteis e, foram previamente estudados por outros autores, na determinação de corantes dispersos, os quais foram quantificados em concentrações entre 50 e $3452 \mathrm{ng} \mathrm{L}^{-1}$. $^{6-8}$

As coletas foram realizadas utilizando frascos de vidro âmbar previamente limpos e descontaminados. O transporte das amostras foi realizado de acordo com o Guia Nacional de Coleta e Preservação de Amostras da Companhia Ambiental do Estado de São Paulo (CETESB). ${ }^{25}$ Em cada ponto, foi coletado $1 \mathrm{~L}$ de amostra e, no laboratório foram imediatamente filtradas utilizando um sistema de filtração a vácuo, utilizando um pré-filtro com porosidade $1,2 \mu \mathrm{m}$. Em seguida, as amostras foram extraídas empregando LLE e os extratos secos foram preservados a $4{ }^{\circ} \mathrm{C}$ em refrigerador até as análises cromatográficas.

\section{RESULTADOS E DISCUSSÃO}

\section{Análise instrumental}

O uso da cromatografia líquida é importante para a separação do corante de interesse de outros interferentes presentes na amostra, uma vez que o efeito matriz pode suprimir o espectro de absorção do corante se medido diretamente da amostra.

Foram analisadas diferentes proporções de solvente na eluição por gradiente, entretanto, não foi possível separar os dois compostos de interesse (Solvent Yellow 34 e Basic Yellow 2). Por apresentarem o mesmo tempo de retenção e o mesmo espectro de absorção UV por HPLC/DAD (Figura 2), os corantes foram quantificados nas amostras ambientais como auramina total.

Usando os parâmetros como tempo de retenção $\left(t_{R}\right)$, fator constante de retenção $(k)$ e resolução entre os picos cromatográficos obteve-se como a melhor condição de eluição uma corrida isocrática com fase móvel metanol/água acidificada $(0,1 \%) 50: 50$ (v/v), a qual garante maior estabilidade nas condições de análise considerando inclusive, o clean-up, requerido para a determinação dos corantes auramina em matrizes aquáticas complexas como são as amostras de águas superficiais. Além disso, a intensidade do pico cromatográfico dos corantes auramina foi maior com o uso do metanol como fase móvel (Figura 3S, Material Suplementar).

O tempo total de cada análise a uma vazão de fase móvel de $0,8 \mathrm{~mL} \mathrm{~min}^{-1}$ foi de 10 minutos. Os cromatogramas foram avaliados em $437 \mathrm{~nm}$ (comprimento de onda de máxima absorção dos corantes analisados) e a identificação do pico foi baseada no espectro de absorção e no tempo de retenção de 7,66 \pm 0,42 minutos (Figura 2).

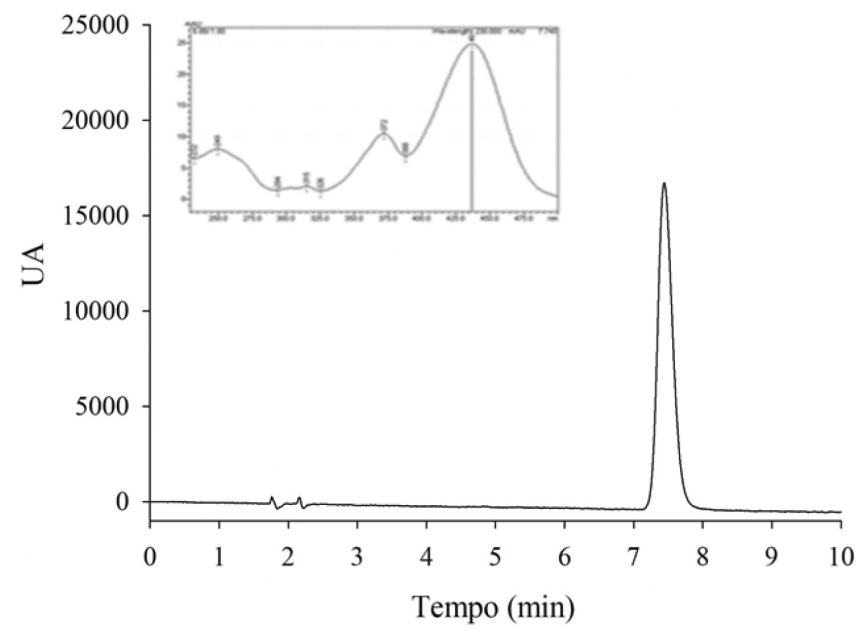

Figura 2. Cromatograma e espectro de absorção $(\lambda=437 \mathrm{~nm})$ do corante auramina (solução padrão em concentração de $10 \mu \mathrm{g} \mathrm{L}^{-1}$ ) obtidos por HPLC/ $D A D\left(t_{r}=7,66 \pm 0,42\right.$ minutos) empregando uma coluna de fase reversa C18 e fase móvel isocrática (metanol/água acidificada $(0,1 \%) 50: 50(\mathrm{v} / \mathrm{v})$ ) a vazão de $0,8 \mathrm{~mL} \mathrm{~min}^{-1}$

\section{Preparo de amostra}

Dois métodos de preparo de amostras foram avaliados, LLE e SPE. A porcentagem de recuperação e o coeficiente de variação obtidos para os três níveis de concentrações (baixo: próximo ao LQI; médio: 2x o nível baixo; alto: 10x o nível mais baixo) foram usados para avaliar a exatidão e precisão dos métodos (Tabela 1). A extração líquido-líquido apresentou maior recuperação dos analitos, superiores a $80 \%$ para os três níveis de concentrações. A extração em fase sólida, por sua vez, apresentou variações nas porcentagens de recuperação para os diferentes níveis (35-73\%). Já os coeficientes de variação que expressam a repetibilidade como uma medida da precisão do método analítico variaram entre 0,4 e $18 \%$ para as diferentes concentrações e os dois métodos de extração, exceto para análise por LLE do menor nível de concentração $\left(0,5 \mu \mathrm{g} \mathrm{L} \mathrm{L}^{-1}\right)$. De forma geral, os menores $\mathrm{CV}$ foram obtidos para a extração por SPE o qual pode estar associado a menor vulnerabilidade a erros aleatórios durante a etapa de preparo de amostras quando comparado à LLE, entretanto, foi possível obter CV satisfatórios em ambas as técnicas de preparo de amostras avaliadas. A aceitabilidade destes valores será discutida no item validação de método analítico.

\section{Validação do método analítico por LLE e HPLC/DAD}

Considerando os resultados descritos nos itens anteriores, optouse por adotar o método clássico por extração líquido-líquido e HPLC/ DAD para determinação do corante auramina em amostras de águas superficiais, bem como em amostras dos testes de ecotoxicidade, 
Tabela 1. Porcentagem de recuperação e coeficiente de variação (CV) dos dois métodos de extração utilizados para a determinação do corante auramina avaliado em triplicata em três diferentes concentrações

\begin{tabular}{|c|c|c|c|c|}
\hline \multirow{2}{*}{ Nível } & \multicolumn{2}{|c|}{ Extração líquido-líquido } & \multicolumn{2}{|c|}{ Extração em fase sólida } \\
\hline & Recuperação ${ }^{\mathrm{a}}(\%)$ & $\mathrm{CV}(\%)$ & Recuperaçãoa $(\%)$ & $\mathrm{CV}(\%)$ \\
\hline Baixo $\left(0,5 \mu \mathrm{g} \mathrm{L}^{-1}\right)$ & $82(74-110)$ & 29 & $35(34,7-34,9)$ & 0,4 \\
\hline Médio $\left(1,0 \mu \mathrm{g} \mathrm{L}^{-1}\right)$ & $90(83-99)$ & 8,9 & $37(30-43)$ & 18 \\
\hline Alto $\left(5,0 \mu \mathrm{g} \mathrm{L}^{-1}\right)$ & $87(83-93)$ & 5,6 & $73(69-77)$ & 7,1 \\
\hline
\end{tabular}

${ }^{a}$ Valores apresentados como média e intervalo de recuperação entre parênteses.

portanto, as seguintes figuras de mérito foram estudadas para avaliar a aceitabilidade do método analítico escolhido.

\section{Seletividade}

A seletividade do método foi avaliada pela comparação entre os cromatogramas obtidos para (i) branco: água ultrapura isenta dos analitos de interesse; (ii) amostra: água superficial isenta dos analitos de interesse; (iii) água fortificada ou amostra sintética: água ultrapura na qual foram adicionados $10 \mu \mathrm{g} \mathrm{L}^{-1}$ do corante auramina e, (iv) padrão: solução padrão $10 \mu \mathrm{g} \mathrm{L}^{-1}$ do corante auramina preparada em uma solução de mesma composição da fase móvel empregada na análise cromatográfica. Diferente das outras três, a solução padrão foi injetada diretamente no HPLC/DAD, enquanto, que o branco, a amostra e a água fortificada foram submetidas à todas as etapas do preparo de amostras antes da análise por HPLC/DAD. A Figura 3 mostra que apenas o sinal referente aos corantes de interesse está presente no tempo de retenção esperado e não há sinal correspondente a interferentes, indicando a seletividade do método proposto, uma vez que foi possível distinguir a resposta do corante em função da presença de possíveis interferentes.

\section{Linearidade instrumental}

A curva de calibração obtida possui relação linear satisfatória, com valor de coeficiente de determinação $\left(R^{2}\right)$ superior a 0,990 (Figura 4a). Além disso, o gráfico de resíduos (Figura 4b) da curva analítica apresentou uma distribuição aleatória em torno da linha reta, como recomendado pelo INMETRO. Em relação à faixa de trabalho, um nível de significância de $5 \%$ foi considerado, dessa forma, os pontos que não atendiam a esse critério foram desconsiderados, limitando a faixa de trabalho até $100 \mu \mathrm{g} \mathrm{L}^{-1}$.

Limites de detecção e de quantificação

Os limites de detecção e quantificação foram obtidos instrumentalmente (LDI e LQI) (por meio da curva analítica) e para o método analítico (LD e LQ), após considerar os fatores

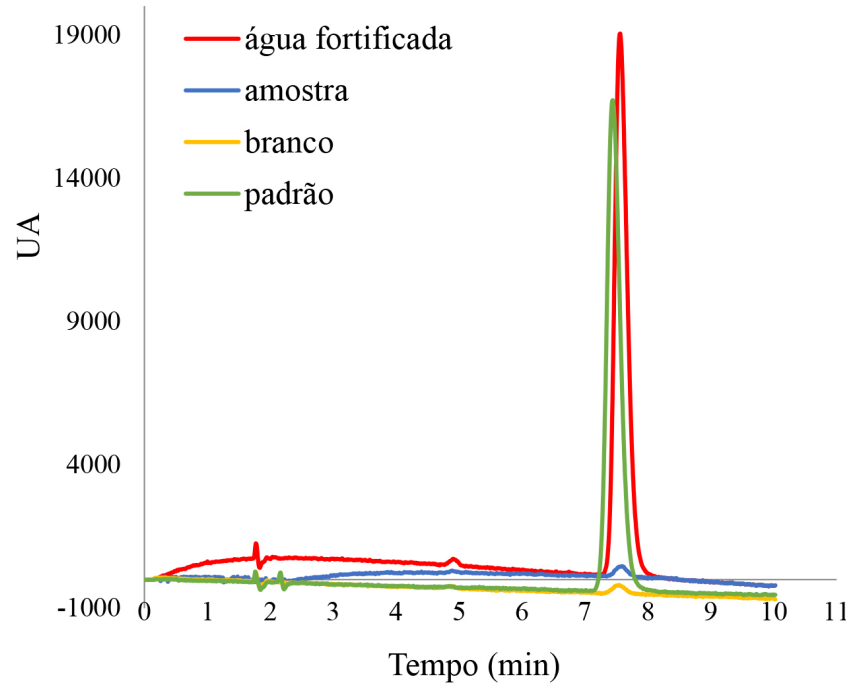

Figura 3. Cromatogramas obtidos por HPLC/DAD ( $\lambda=437 \mathrm{~nm})$ utilizados $\mathrm{na}$ avaliação da seletividade: Água fortificada (amostra ultrapura contendo o corante auramina em concentração de $10 \mu \mathrm{g} L^{-1}$ e extraída por LLE); Amostra (água superficial isenta do analito de interesse e analisada por LLE e HPLC/ $D A D$ ); Branco (água ultrapura isenta do analito de interesse e analisada por LLE e HPLC/DAD) e Padrão (solução padrão do corante auramina em concentração de $10 \mu \mathrm{g} \mathrm{L}^{-1}$ )

de concentração/diluição empregados nas etapas de preparo de amostras. Os valores de LDI e LQI foram de 3,6 e 12,1 $\mu \mathrm{g} \mathrm{L}^{-1}$, respectivamente. Esses valores foram considerados adequados para a quantificação dos corantes nos testes de ecotoxicidade sem a necessidade da etapa prévia de extração, uma vez que estão abaixo de todas as concentrações testadas. Porém, em situações nas quais as concentrações testadas foram inferiores ao LQI, a etapa de preparo de amostras empregando LLE ou SPE se torna fundamental para a determinação da concentração real dos testes ecotoxicológicos. Por
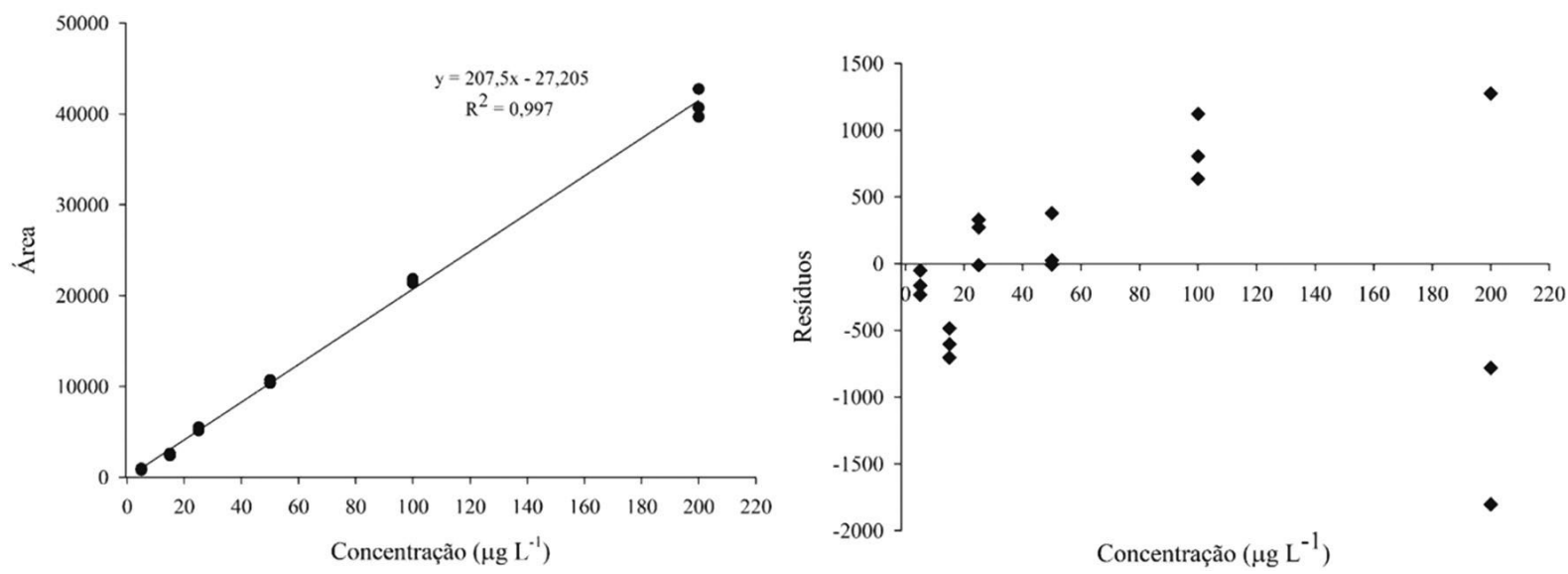

Figura 4. Curva analítica obtida por HPLC/DAD (a esquerda) e gráfico de resíduos (a direita) para análise do corante auramina 
outro lado, concentrações maiores que $100 \mu \mathrm{g} \mathrm{L}^{-1}$ necessitam de uma etapa prévia de diluição atendendo as limitações da faixa de trabalho da curva analítica estabelecida.

Em relação a detectabilidade do método analítico empregando LLE e HPLC/DAD, com um fator de concentração de 1000 vezes, foram obtidos valores de 3,6 e $12 \mathrm{ng} \mathrm{L}^{-1}$ para $\mathrm{LD}$ e $\mathrm{LQ}$, respectivamente.

Lepom e colaboradores recomendam que os métodos analíticos empregados na quantificação de contaminantes ambientais destinados a uma avaliação de risco apresentem $L Q<30 \%$ do critério de proteção ambiental derivado. ${ }^{26}$ Valores de PNEC para os corantes auramina estão descritos na literatura ${ }^{15}$ como 0,92 e $4,6 \mu \mathrm{g} \mathrm{L}^{-1}$, para Solvent Yellow 34 e Basic Yellow 2, respectivamente. Sendo assim, os valores de LD e LQ obtidos também são considerados satisfatórios para fins de monitoramento ambiental, pois estão cerca de 75 vezes menores que os critérios de proteção ambiental derivados para ambos os corantes.

\section{Precisão instrumental}

As precisões instrumentais obtidas para as análises dos corantes por HPLC/DAD em três diferentes concentrações foram consideradas satisfatórias, com coeficientes de variação de 5,5\%, 4,3\% e 2,4\% para as concentrações de 25,50 e $100 \mu \mathrm{g} \mathrm{L}^{-1}$, respectivamente, para repetitividade $(n=6)$ obtidas pelas medidas das áreas dos picos cromatográficos (Tabela 3S, Material Suplementar).

\section{Exatidão e precisão do método analítico}

O método proposto por LLE e HPLC/DAD mostrou ser eficiente para a determinação dos corantes auramina, considerando a exatidão com porcentagem da média da recuperação variando de 82 a $90 \%$ e a precisão com CV de 5,6 a 29\% (Tabela 1). O CV para o nível baixo $\left(0,5 \mu \mathrm{g} \mathrm{L^{-1 }}\right)$ foi um pouco mais alto, quando comparados aos outros níveis, porém, de acordo com as recomendações do INMETRO, para concentrações abaixo de $1,0 \mu \mathrm{g} \mathrm{L}^{-1}$, precisões com $\mathrm{CV}$ de até $30 \%$ são aceitáveis. A ANVISA ${ }^{22}$ não estabelece nenhum valor de aceitação, devendo ser definidos de acordo com o objetivo do método, a variabilidade intrínseca do método, concentração de trabalho e a concentração do analito na amostra. Os critérios de aceitação tanto para precisão, quanto para recuperação obtidos neste trabalho estão de acordo com os recomendados. ${ }^{21}$

Após avaliar a recuperação dos corantes simultaneamente e determinar o melhor método de extração, as recuperações dos analitos foram analisadas separadamente por LLE e HPLC/DAD, obtendo exatidões superiores a $80 \%$ para as seguintes amostras analisadas: (i) soluções individuais dos corantes Solvent Yellow 34 e Basic Yellow 2 e, (ii) uma solução contendo os dois corantes (Figura 4S, Material Suplementar). As soluções individuais continham concentrações de $1,0 \mu \mathrm{g} \mathrm{L}^{-1}$ dos corantes e no processo de extração as mesmas foram concentradas 1.000 vezes $(400 \mathrm{~mL}$ de amostra para $0,4 \mathrm{~mL}$ de extrato final). Isso tem relevância, quando se considera a aplicação do método para determinação nominal de testes ecotoxicológicos, as quais podem ser realizadas separadamente ou em misturas. No entanto, quando considerada a aplicação do método para amostras de águas superficiais, a ocorrência destes corantes deverá ser reportada como auramina total. Além disso, em se tratando de um par ácidobase de Bronsted, a espécie química predominante é dependente do $\mathrm{pH}$ do meio, uma vez que $\mathrm{pH}$ com duas unidades abaixo do pKa garantiria 99\% da espécie protonada; $\mathrm{pH}$ com duas unidades acima do pKa $(10,12)$ garantiria $99 \%$ da espécie desprotonada. Nas amostras analisadas, o pH variou de 6,9 a 7,8, dessa forma, o corante predominante seria o Basic Yellow 2. Devido a isso, uma amostra do Rio Piracicaba foi fortificada com solução contendo $1,0 \mu \mathrm{g} \mathrm{L}^{-1}$ do corante auramina na sua forma protonada (Basic Yellow 2) e submetida ao processo de extração por LLE, no qual foi obtido uma recuperação de $89 \%$.

\section{Aplicação do método}

\section{Testes de ecotoxicidade}

O teste foi devidamente validado e os resultados obtidos podem ser consultados no material suplementar (Tabela 4S). Com os limites de detecção e quantificação instrumentais obtidos, o método desenvolvido mostrou ser eficiente na determinação das concentrações reais dos corantes nos testes de ecotoxicidade, pois apresentou um LQI menor que as concentrações testadas. Dessa forma, foi descartada a necessidade de concentração dos analitos das amostras por técnicas de extração (LLE ou SPE). Para a quantificação das concentrações de exposição no teste uma nova curva analítica foi construída (Tabela 5S, Material Suplementar).

Antes da injeção das amostras do teste de ecotoxicidade no equipamento, diluiram-se as mesmas em soluções semelhantes a fase móvel do método analítico, metanol/água (50:50) e os valores das concentrações medidas foram obtidos, multiplicando pelo fator de diluição utilizado (Figura 5S, Tabela 6S, Material Suplementar). Como podemos observar na Figura $5 \mathrm{~S}$ todas as concentrações medidas ficaram bem próximas das nominais, permanecendo dentro da faixa aceitável que é de 80-120\%.

\section{Amostras ambientais}

Um total de 9 amostras foram analisadas por LLE e HPLC/ DAD em 3 campanhas amostrais. Na extração, o fator de diluição utilizado foi de 1.000 vezes (1 litro de amostra, em $1 \mathrm{~mL}$ de solução extrato contendo metanol/água (50:50)). Em todas as campanhas as concentrações dos corantes auramina estiveram abaixo do limite de detecção do método analítico $\left(3,6 \mathrm{ng} \mathrm{L}^{-1}\right)$. O perfil cromatográfico de cada ponto amostral analisado neste trabalho pode ser consultado no Material Suplementar (Figura 6S).

Uma vez que o LD é inferior as concentrações dos corantes auramina considerada segura para a biota aquática, ${ }^{15}$ pode-se sugerir que esses compostos não apresentam um potencial risco para a vida aquática nas amostras investigadas neste trabalho. Entretanto, para realmente inferir um risco ambiental dos corantes auramina nos corpos d'água, um monitoramento mais amplo deve ser realizado.

O método analítico desenvolvido foi aplicado em um cenário considerado crítico de contaminação por corantes, pois a cidade de Americana-SP possui o principal polo têxtil do Brasil, responsável por $85 \%$ da produção de tecidos do país. Apesar dos corantes auramina serem utilizados no tingimento de tecido, os mesmos também são aplicados para tingir papéis. Considerando a grande importância destes corantes, pesquisadores de diversos lugares do mundo poderão investigar a ocorrência dos corantes auramina em diferentes cenários de possível contaminação empregando um método simples e barato, que também poderá ser adaptado para métodos multiresíduos (empregando SPE como preparo de amostras), para então verificar se existe um potencial risco destes compostos para a vida aquática em outros rios.

Alguns autores também avaliaram o risco ambiental relacionado ao descarte de corantes nos rios analisados neste trabalho (Rio Piracicaba e Ribeirão Quilombo). Vacchi e colaboradores ${ }^{7}$ detectaram concentrações que variaram de 50 à $500 \mathrm{ng} \mathrm{L}^{-1}$ do corante Disperse Red 1, verificando um risco para a biota aquática decorrente das concentrações determinadas deste corante. Outro trabalho, por sua vez, quantificou dois novos fenilbenzotriazóis, não-Cl PBTA-9 e PBTA-9, derivados do corante Disperse Violet 93, nos mesmos pontos amostrais e a avaliação de risco preliminar não indicou potencial risco de ambos compostos para a vida aquática. ${ }^{27}$ 


\section{CONCLUSÕES}

O método desenvolvido foi aplicado com sucesso na determinação dos corantes auramina em amostras de águas superficiais e em testes de ecotoxicidade, apresentando resultados aceitáveis para seletividade, linearidade, precisão e exatidão, além de limites de detecção e quantificação apropriados para ambas aplicações. O método analítico apresentou sensibilidade adequada para detectar concentrações 75 vezes menores que o PNEC derivado para esses corantes na literatura e para analisar as amostras dos testes de ecotoxicidade sem a necessidade da etapa de extração. Em todas as amostras ambientais analisadas, os corantes auramina estiveram abaixo do LD do método analítico $\left(3,6 \mathrm{ng} \mathrm{L}^{-1}\right)$, portanto, sugere-se que não há um risco potencial relacionado à presença desses corantes nas amostras investigadas. Finalmente, os resultados indicaram que o método proposto foi eficaz e prático para a análise de traços desses contaminantes ambientais em matrizes aquáticas e será uma ferramenta importante para verificar o potencial risco dos corantes auramina em trabalhos futuros.

\section{MATERIAL SUPLEMENTAR}

No material suplementar, disponível em http://quimicanova.sbq. org.br, em arquivo pdf e com acesso livre, encontram-se os resultados do planejamento experimental realizado para otimização do preparo de amostras empregando extração líquido-líquido, bem como detalhes sobre os pontos amostrais estudados e dados complementares da validação do método e da quantificação dos corantes auramina nos meios de cultivo e nas amostras ambientais utilizando HPLC/DAD.

\section{AGRADECIMENTOS}

O presente trabalho foi realizado com apoio da Coordenação de Aperfeiçoamento de Pessoal de Nível Superior - Brasil (CAPES) - Código de Financiamento 001. As autoras agradecem à FAPESP (proc. 2014/24749-6) pelo auxílio financeiro ao projeto e ao PIBIC pela bolsa de iniciação científica de M. K. Kasahara.

\section{REFERÊNCIAS}

1. Guaratini, C. C. I.; Zanoni, M. V. B.; Quim. Nova 2000, 23, 71.

2. Zanoni, M. V. B.; Yamanaka, H.; Caracterização química, toxicológica, métodos de detecção e tratamento, Ed. Cultura Acadêmica: São Paulo, 2016.

3. Leite, L. S.; Maselli, B. S.; Umbuzeiro, G. A.; Pupo, R. F.; Chemosphere 2016, 148, 511.

4. Umbuzeiro, G. A.; Freeman, H. S.; Warren, S. H.; de Oliveira, D. P.; Terao, Y.; Watanabe, T.; Claxton, L. D.; Chemosphere 2005, 60, 55.

5. Carneiro, P. A.; Umbuzeiro, G. A.; Oliveira, D. P.; Zanoni, M. V. B.; J. Hazard. Mater. 2010, 174, 694.

6. Zocolo, G. J.; Pilon dos Santos, G.; Vendemiatti, J.; Vacchi, F. I.; Umbuzeiro, G. de A.; Zanoni, M. V. B.; J. Chromatogr. Sci. 2015, 1.
7. Vacchi, F. I.; Von der Ohe, P. C.; Albuquerque, A. F.; Vendemiatti, J. A. S.; Azevedo, C. C. J.; Honório, J. G.; Silva, B. F.; Zanoni, M. V. B.; Henry, T. B.; Nogueira, A. J.; Umbuzeiro, G. A.; Chemosphere 2016, $156,95$.

8. Vacchi, F. I.; Vendemiatti, J. A. S.; Ferreira, B.; Silva, D.; Valnice, M.; Zanoni, B.; Umbuzeiro, G. A.; Sci. Total Environ. 2017, 601-602, 230.

9. Maguire, R. J.; Water Sci. Technol. 1992, 26, 265.

10. Rajaguru, P.; Vidya, L.; Baskarasethupathi, B.; Kumar, P. A.; Palanivel, M.; Kalaiselvi, K.; Mutat. Res. 2002, 517, 29.

11. Gessner, T.; Mayer, U.; Em Ullmann's Encyclopedia of Industrial Chemistry Wiley-VCH Verlag GmbH \& Co. KGaA: Weinheim, Germany, 2000.

12. Tkaczyk, A.; Mitrowska, K.; Posyniak, A.; Sci. Total Environ. 2020, 717, 137222.

13. Industrial Dyes: Chemistry, Properties, Applications; Hunger, K., ed.; Wiley: Hoboken, 2003, 648 p.

14. IARC, IARC Monographs on the Evaluation of Carcinogenic Risks to Humans, Vol. 99 - Some Aromatic Amines, Organic Dyes, and Related Exposures, 2010, available at https://monographs.iarc.fr/ENG/ Monographs/vo199/mono99.pdf, accessed at March 2021.

15. Azevedo, C. C. J.; de Oliveira, R.; Suares-Rocha, P.; Sousa-Moura, D.; Li, A. T.; Grisolia, C. K.; Umbuzeiro, G. A.; Montagner, C. C.; Environ. Sci. Pollut. Res. 2020, 28, 1866.

16. EU-JRC, Technical Guidance Document on Risk Assessment in support of Commission Directive 93/67/EEC on Risk Assessment for new notified substances Commission Regulation (EC) No 1488/94 on Risk Assessment for existing substances Directive 98/8/EC of the European office for official publications of the european communities, Luxembourg, 2003.

17. SBMCTA, Protocolo para Derivação de Critérios de Qualidade da Água para proteção da Vida Aquática no Brasil, 2011.

18. Moermond, C. T. A.; Kase, R.; Korkaric, M.; Ågerstrand, M.; Environ. Toxicol. Chem. 2016, 35, 1297.

19. OECD, Guidelines for the testing of Chemicals. Freshwater Alga and Cyanobacteria, Growth Inhibition Test, 2011, available at https://doi. org/10.1787/9789264203785-en, accessed at March 2021.

20. Parrott, J. L.; Bartlett, A. J.; Balakrishnan, V. K.; Environ. Pollut. 2016, $210,40$.

21. INMETRO, Orientações sobre Validação de Métodos de Ensaios Químicos, 2016.

22. ANVISA, Resolução da Diretoria Colegiada, RDC n ${ }^{\circ}$ 166, 2017.

23. OECD, Test No. 211: Daphnia magna Reproduction Test, 2012, available at https://doi.org/10.1787/9789264185203-en, accessed at March 2021.

24. Jasi, A.; Paraszkiewicz, K. In Microbial Degradation of Synthetic Dyes in Wastewaters; Singh, S. N., ed.; Springer: New York, 2017.

25. Agência Nacional de Águas e Companhia Ambiental do Estado de São Paulo, CETESB, 2011.

26. Lepom, P.; Brown, B.; Hanke, G.; Loos, R.; Quevauviller, P.; Wollgast, J.; J. Chromatogr. A 2009, 1216, 302.

27. Vendemiatt, J. A. S.; Camparotto, N. G.; Vidal, C.; Cristale, J.; Souza, J. A.; Montagner, C. C.; Umbuzeiro, G. A.; Prediger, P.; J. Hazard. Mater. 2021, 403, https://doi.org/10.1016/j.jhazmat.2020.12373. 